\title{
Candida balanitis. Clinical and mycological study about the efficacy of a single-day oral treatment with itraconazole (400 mg)
}

\author{
Alexandro Bonifaz,2, Andrés Tirado-Sánchez,3, Cristina Jaramillo-Manzur, \\ Javier Araiza1,2, Leonel Fierro-Arias ${ }^{1}$
}

${ }^{1}$ Department of Dermatology, Hospital General de México. "Dr. Eduardo Liceaga", Mexico City, Mexico, ${ }^{2}$ Department of Mycology, Hospital General de México. "Dr. Eduardo Liceaga”, Mexico City, Mexico, 3Department of Internal Medicine, Hospital General de México. "Dr. Eduardo Liceaga”, Mexico City, Mexico

Corresponding author: Prof. Alexandro Bonifaz, E-mail: a_bonifaz@yahoo.com.mx

\begin{abstract}
Objective: To assess the efficacy of a single dose of itraconazole $(400 \mathrm{mg} /$ day), divided into two doses in the treatment of Candida balanitis. Material and Methods: We carried out a prospective, non-compared study in patients with clinically verified Candida balanitis and through direct examinations, stains and cultures. Each of the patients was given a treatment scheme of itraconazole $400 \mathrm{mg} /$ day, divided into two doses. Revisions were carried out at baseline and subsequently on days 3, 7, and 15. Results: We included 26 cases, with an average age of 43.5 years. A predominance of Candida albicans was obtained in $69.2 \%$ and the remaining were Candida non-albicans species. At the end of the treatment and follow-up for 7 days, clinical and mycological cure was obtained in 22/26 patients (84.6\%). Side effects were presented in two patients $(7.7 \%)$, one with moderate dyspepsia and the other with moderate headache. Conclusion: The treatment of Candida balanitis with a single dose of $400 \mathrm{mg}$ of oral itraconazole is effective, well tolerated and represents a new therapeutic alternative of short duration.
\end{abstract}

Key words: Balanitis; Candida albicans; Candida no-albicans; Itraconazole; Treatment

\section{INTRODUCTION}

Balanitis caused by several species of Candida or Candida balanitis (CB), is an opportunistic mycosis that is observed with relative frequency, representing $11 \%$ of sexually transmitted diseases (STD) [1-3], and $30-35 \%$ of the infectious balanitis [4,5]. It is commonly observed in acute or subacute form (5-7 days) and usually affects young adults. CB clinically manifests in the form of a balanitis or balanopostitis, affecting the mucosa of the penis, particularly glans, body of the penis (balanitis) and balanoprepucial zone (balanopostitis), as erythematous plaques often with micropustules and whitish plaques, which over time evolvedto erythematous plaques with erosions and fissures. Patients initially refer moderate to severe itching and subsequently burning $[2,6]$.
$\mathrm{CB}$ is a classic opportunistic infection caused mainly by Candida albicans, but also by other Candida spp. Its presence is closely related to vulvovaginitis and most cases are influenced by sexual activity in women who have the disease or simply, have an increased microbiota of Candida sp. Other risk factors include: diabetes mellitus; chronic use of topical and systemic corticosteroids, as well as immunosuppression $[2,6,7]$.

Diagnosis of CB is eminently clinical and should be corroborated with mycological tests $[4,6,8]$. The treatment of CB include one of the following options: Topical treatments include nystatin or imidazolic derivatives such as clotrimazole, ketoconazole, or fenticonazole. However, though the mentioned treatment is particularly useful, the time of therapy is longer and thus, the decrease in symptoms and

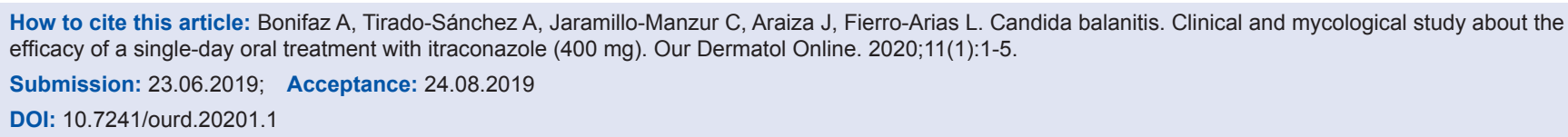


signs is slower and some authors have reported significant local irritation [1]. Fluconazole is a triazole derivative and the most effective and fastresponse oral antifungal, and it is considered to be the treatment of choice according to the treatment guidelines $[6,7]$.

This study aims to assess the efficacy of a single dose of itraconazole $(400 \mathrm{mg} /$ day $)$, divided into two doses in the treatment of $\mathrm{CB}$.

\section{MATERIAL AND METHODS}

In this prospective and non-controlled study, we included uncircumcised patients with balanitis and balanopostitis caused by Candida species. All patients gave their informed consent before they were admitted to the study. CB was diagnosed clinically and by the following mycological studies: Direct examinations with $\mathrm{KOH}(10 \%)$ and blue cotton, observing pseudohyphae and blastoconidia or only abundant blastoconidia. Isolation in two culture media: Sabouraud dextrose agar, BiggyNickerson (Difco Co ${ }^{\circledR}$ ) and CHROMagar-Candida ${ }^{\circledR}$. The identification of Candida species was made by the reading of the second culture medium and confirmed by the degradation of carbohydrates by the commercial biochemical method of API-yeast- $20^{\circledR}$ and confirmed by the proteomic method of MALDI-TOF MS (VITEK2 $\left.{ }^{\circledR}\right)[4,8,9]$.

The treatment scheme was a single dose of $400 \mathrm{mg}$ of oral itraconazole, distributed into two doses: $200 \mathrm{mg}$ after breakfast and $200 \mathrm{mg}$ after dinner. Clinical reviews and mycological studies were carried out: at baseline and on days 3, 7, and 15 after medication. During the follow-up time, the patients receive any topical or oral antifungal and those patients who presented signs and symptoms of balanitis at visit 3 (7 days after medication), and positive mycological study (yeasts and pseudohyphae), were considered therapeutic failures and continued with another therapy.

\section{Statistical Analysis}

Clinical and mycological data were analyzed by using chi-square test to compare the results of treatment during control visits. Fisher exact test was used when expected values were less than 5. Statistical significance was set at $\mathrm{P}=<0.001$. SPSS version 23 for Windows was used for analysis.

\section{RESULTS}

Twenty-six cases of balanitis and balanoposthitis caused by several Candida species were included. The main demographic and mycological data of the study is presented in Table 1.

In all cases, the response to treatment was evaluated at baseline and subsequent visits (3,7, and 15 days). The results were the following: At visit 2 ( 3 days after treatment), three patients showed signs or symptoms of CB and tested positive for mycological studies. At visit 3 (7 days of follow-up), one more patient presented clinical and mycological activity. At visit 4 (15 days of follow-up), only 19 patients attended to the evaluation, of which none had signs or symptoms of $\mathrm{CB}$, and direct examinations and cultures were negative (Table 2).

The clinical cure of balanitis was observed from 3 days of use of itraconazole, at 15 days of management with itraconazole efficacy was in $100 \%$ of cases (Table 2). In Table 3, it is mentioned that the mycological cure

\begin{tabular}{lc} 
Table 1: Main demographic and mycological data of the study \\
\hline Male patients & $\mathbf{2 6}$ cases \\
\hline Age (years), range. & $43.5(18-72)$ \\
Disease evolution (days), range. & $5.6(2-18)$ \\
Predisposing factors, (\%) & \\
After sexual contact. & $15(57.7)$ \\
Topical corticosteroid use & $4(15.38)$ \\
Type 2 diabetes mellitus & $3(11.53)$ \\
After sexual contact + typo 2 DM & $1(3.84)$ \\
None identified & $3(11.53)$ \\
Direct examination (\%) & \\
Pseudohyphae and blastoconidia & $22(85)$ \\
Only blastoconidia & $4(15)$ \\
Etiologic agents (\%) & \\
Candida albicans & $18(69.23)$ \\
Candida glabrata & $4(15.38)^{*}$ \\
Candida tropicalis & $2(7.69)$ \\
Candida krusei & $1(3.84)$ \\
C. albicans + C. glabrata & $1(3.84)$ \\
\hline
\end{tabular}

Table 2: Clinical efficacy of itraconazole in patients with Candida balanitis

\begin{tabular}{lccc}
\hline Clinical examination & $\mathbf{3}$ days $(\%)^{*}$ & $\mathbf{7}$ days $(\%)^{*}$ & $\mathbf{1 5}$ days $(\%)^{*}$ \\
\hline Cure & $23(88.5)$ & $22(85)$ & $19(100)$ \\
Failure & $3(11.5)$ & $4(15)$ & 0 \\
Total & $26(100)$ & $26(100)$ & $19(100)$ \\
\hline${ }^{*} \mathrm{X}^{2}, \mathrm{P}=\leq 0.01$ & & &
\end{tabular}

Table 3: Mycological efficacy of itraconazole in patients with Candida balanitis.

\begin{tabular}{lccc}
\hline Mycological examination & $\mathbf{3}$ days (\%) & $\mathbf{7}$ days (\%) & $\mathbf{1 5}$ days (\%) \\
\hline Candida albicans & $16(89)$ & $16(89)$ & $13(68.4)$ \\
Candida non-albicans & 6 & 6 & $5(31.6)$ \\
Total & $22(100)$ & $22(100)$ & $18(100)$ \\
\hline
\end{tabular}

Mixed case was excluded for analysis. ${ }^{*}$ Fisher, $\mathrm{P}=\leq 0.01$. 
of balanitis in Candida albicans species was observed from 3 days of use of itraconazole in $73 \%$ of the cases, maintaining this efficacy at 15 days of management. In Candida non-albicans species was $27 \%$ in 3 weeks, not modified at 15 days of treatment.

The four patients who failed treatment had the following predisposing factors: 2 had previous treatments with topical steroids and the other two had type 2 diabetes mellitus. Three of the therapeutic failure cases were caused by C. albicans and one case by C. tropicalis. The rest of the etiological agents, included the mixed case, presented therapeutic success (Table 3). Side effects were present in two patients $(7.7 \%)$. One patient showed moderate dyspepsia and one had a moderate headache. None of the patients required additional treatment.

\section{DISCUSSION}

Clinically, balanitis and balanoposthitis can present in a similar way. Most of them are caused by yeasts, especially Candida spp. Some reports indicate that in a third of the cases, these are caused by bacteria, especially b-hemolytic Streptococcus, Staphylococcus aureus, Pseudomonas sp., and Gardnerella vaginalis, among others $[1,5,10-13]$.

Mycotic balanitis is frequently caused by C. albicans and, to a lesser extent, by other Candida species, and exceptionally by other yeasts such as: Rhodotorula mucilaginosa and Saccharomyces cerevisiae $[6,7,12,13]$. Malassezia spp. have also been reported. Its role is like normal microbiota of the preputial space and probably influences the condition $[6,13,14]$. CB is an infection that is directly related to Candida vulvovaginitis (VVC) due to sexual relations (vaginal, anogenital, and urogenital) and other predisposing factors $[1-3,15,16]$. In general, $\mathrm{CB}$ is a superficial infection, of a rapid course and with few complications, but occasionally, it can cause urethritis [16].

In $\mathrm{CB}$, systemic antifungals are the main choice of treatment. The advantage of oral therapy over topical therapy is that it is simpler and faster than the second, leading to a faster decrease in signs (erythema and fissures) and symptoms (itching and burning) $[2,12,17,18]$.

The first oral antifungal that showed great impact on genital candidiasis was ketoconazole. Its use is currently limited due to side effects and drug interactions $[6,19]$.
Subsequently, triazole derivatives emerged with a higher spectrum indicated for mycosis and exhibiting lower MICs against the different species, allowing the shortening of the treatment schemes [18,19].

The first short-treatment scheme for genital candidosis (VVC and CB) was fluconazole, often used in adults at a single $150 \mathrm{mg}$ dose. It obtained a high rate of therapeutic response. In some studies, the cure percentage was 92\% [18]. Moreover, the European guidelines for the management of $\mathrm{CB}$ considered that fluconazole is the first-line treatment for CB [7]. However, one of the drawbacks of the indiscriminate use of fluconazole is related to the high resistance acquired by various strains of C. albicans, and nonalbicans species, especially Candida krusei, which has developed an intrinsic resistance, and C. glabrata, which has developed an acquired resistance $[6,7,19]$. Despite the fact that fluconazole and itraconazole are two triazolic derivatives, they have not been shown to share the resistance to the triazole ring [20].

Our study aims to determine the efficacy of a single $400 \mathrm{mg}$ dose of itraconazole for the management of CB. This scheme has been tested with good results in cases of VVC and in CB isolated cases [10,21]. According to our study, the cure rate after three days of follow-up was 88.5 and it was $85 \%$ after 7 days.

In our study, we observed that at three days of treatment, 23/26 patients (88.5\%) showed decreased signs and symptoms of the disease. On the other hand, when a patient developed relapse, this was caused by the same etiologic agent. According to the statistical analysis, it is concluded that both groups of species (C. albicans and C. non-albicans) are cured within 3 days and do not present recurrence 15 days later. The results that we obtained can be compared with those obtained in VVC. Although they are clinically different entities, some reports indicate that results of efficacy can be achieved by more than $95 \%[21,22]$. Spacek et al [23], obtained better results with a single $400 \mathrm{mg}$ dose compared to the dose of $200 \mathrm{mg}$ for three days, with the clinical and mycological cure in $97.1 \%$ and $76.9 \%$ respectively, indicating that the first one is effective in VVC and, according to our study, also in CB.

Sixty-nine percent of our cases were caused by C. albicans and the remaining by non-albicans species. This is similar to the observed results in other studies $[1,2,4,10]$. We observed that the therapeutic failures were presented in three strains of 
C. albicans and one of C. tropicalis, while in the four cases produced by C. glabrata and one by C. krusei, therapeutic successes were obtained in all patients. This is relevant for clinical practice, since the last two species are resistant to fluconazole, and itraconazole is an effective option $[6,19]$.

A possible explanation for the therapeutic failure of the four cases $(15.38 \%)$ is not related to the etiological agent, because itraconazole has a spectrum and MICs are suitable for most species, but to the predisposing factors [20]. Therapeutic failure was observed in two patients who previously used topical steroids, while among the other two cases of failure occurred in diabetic patients, so we recommend that the use of a short $400 \mathrm{mg}$ dose is not enough in cases with a predisposing factor, and it probably requires a prolonged treatment.

There are no comparative studies between itraconazole and fluconazole in CB. However, in the literature, there are reports in VVC [24] pointing to a greater efficacy of itraconazole when compared with fluconazole (93.8\% vs $79.03 \%$ respectively, $P=.008)$. However, Pitsouni et al [25] reported a meta-analysis of controlled and randomized studies, indicating that both drugs are effective without statistically significant differences. A recent study of Hu et al [26] reported a case of penile infection by C. albicans, with proven resistance to fluconazole and terbinafine, and it was successfully treated with itraconazole.

In our trial, we did not perform laboratory studies to evaluate possible drug adverse events, due to the fact that the hematological and hepatic abnormalities are not detected at the dose used [20]. It is usual for other types of mycosis such as onychomycosis or deep mycosis to be managed at higher doses and longer times without presenting alterations. It is important to emphasize that both triazolics (fluconazole and itraconazole) have drug interactions due to the metabolism of cytochrome p-450 (CYP 3A4) [27] however, when administered in a short duration schedule in particular at a single dose, it is difficult to present. The absorption of itraconazole is limited with the use of antacids, and thus its concomitant administration should be avoided [20].

Only two patients presented collateral symptoms $(7.7 \%)$. One patient presented with dyspepsia and the other with moderate headache, both effects have been previously reported [20] and did not require additional treatment.
It is necessary to perform future comparative studies between itraconazole $(400 \mathrm{mg})$ and fluconazole $(150 \mathrm{mg})$ in a short scheme, which would give us a better picture of both drugs in the management of $\mathrm{CB}$.

\section{CONCLUSIONS}

According to the results, we conclude that the use of itraconazole at a dose of $400 \mathrm{mg} /$ day divided into two doses is effective and safe for CB with high cure rates. The treatment of CB should begin with mycological diagnosis and confirmation, identification of the etiological agent, and evaluation and control of possible predisposing factors, as well as include clinical followup after treatment.

\section{Statement of Human and Animal Rights}

All procedures followed were in accordance with the ethical standards of the responsible committee on human experimentation (institutional and national) and with the Helsinki Declaration of 1975, as revised in 2008 .

\section{Statement of Informed Consent}

Informed consent was obtained from all patients for being included in the study.

\section{REFERENCES}

1. Abdennader S, Casin I, Janier M, Zavaro A, Vendeuil MO, Traoré F, et al. Balanitis and infectious agents. A prospective study of 100 cases. Ann Dermatol Venereol. 1995;122: 580-4

2. Edwards S. Balanitis and balanoposthitis: a review. Genitourin Med. 1996;72:155-9.

3. Kalra S, Chawla A. Diabetes and balanoposthitis. J Pak Med Assoc. 2016;66:1039-41.

4. Dockerty WG, Sonnex C. Candidal balano-posthitis: a study of diagnostic methods. Genitourin Med. 1995;71:407-9.

5. Abdullah AN, Drake SM, Wade AA, Walzman M. Balanitis (balanoposthitis) in patients attending a department of genitourinary medicine. Int J STD AIDS. 1992;3:128-9.

6. Nyirjesy P, Sobel JD. Genital mycotic infections in patients with diabetes. Postgrad Med. 2013;125:33-46.

7. Edwards SK, Bunker CB, Ziller F, van der Meijden WI. 2013 European guideline for the management of balanoposthitis. Int J STD AIDS. 2014;25:615-26.

8. Neppelenbroek KH, Seó RS, Urban VM, Silva S, Dovigo LN, Jorge $\mathrm{JH}$, et al. Identification of Candida species in the clinical laboratory: a review of conventional, commercial, and molecular techniques. Oral Dis. 2014;20:329-44.

9. Maldonado I, Cataldi S, Garbasz C, Relloso S, Striebeck P, Guelfand L, et al. Identificación de levaduras del género Candida: los métodos convencionales frente a MALDI-TOF MS. Rev Iberoam Micol. 2018;35:151-4.

10. Lisboa C, Santos A, Azevedo F, Pina-Vaz C, Rodrigues AG. Direct impression on Agar surface as a diagnostic sampling procedure for 
Candida balanitis. Sex Transm Infect. 2010;86:32-5.

11. Lisboa C, Ferreira A, Resende C, Rodrigues AG. Infectious balanoposthitis: management, clinical and laboratory features. Int J Dermatol. 2009;48:121-4.

12. Möhrenschlager M, Ring J, Köhn FM. Diagnosis of major sexually transmitted infections in the doctor's office: ectoparasites and yeasts. MMW Fortschr Med. 2004;16:38,40-5.

13. Oriel JD, Partridge BM, Denny MJ, Coleman JC. Genital yeast infections. Br Med J. 1972;30:761-4.

14. Alsterholm M, Flytström I, Leifsdottir R, Faergemann J, Bergbrant IM. Frequency of bacteria, Candida and Malassezia species in balanoposthitis. Acta Derm Venereol. 2008;88:331-6.

15. Ono F, Yasumoto S. Genital candidiasis. Nippon Rinsho. 2009;67:157-61.

16. Li J, Fan SR, Liu XP, Li DM, Nie ZH, Li F, et al. Biased genotype distributions of Candida albicans strains associated with vulvovaginal candidosis and candida balanoposthitis in China. Clin Infect Dis. 2008;47:1119-25.

17. Stary A, Soeltz-Szoets J, Ziegler C, Kinghorn GR, Roy RB. Comparison of the efficacy and safety of oral fluconazole and topical clotrimazole in patients with candida balanitis. Genitourin Med. 1996;72:98-102.

18. Kinghorn GR, Woolley PD. Single-dose fluconazole in the treatment of Candida albicans balanoposthitis. Int J STD AIDS. 1990;1:366-7.

19. Cernicka J, Subik J. Resistance mechanisms in fluconazole-resistant Candida albicans isolates from vaginal candidiasis. Int J Antimicrob Agents. 2006;27:403-8.

20. Caputo R. Itraconazole (Sporanox) in superficial and systemic fungal infections Expert Rev Anti Infect Ther. 2003;1:531-42.

21. Urünsak M, Ilkit M, Evrüke C, Urünsak I. Clinical and mycological efficacy of single-day oral treatment with itraconazole $(400 \mathrm{mg})$ in acute vulvovaginal candidosis. Mycoses. 2004;47:422-7.

22. Chattopadhyay S. Itraconazole: better choice to treat recurrent vulvovaginal candidoses. J Indian Med Assoc. 2004;102:523.

23. Spacek J, Buchta V. Itraconazole in the treatment of acute and recurrent vulvovaginal candidosis: comparison of a 1-day and a 3-day regimen. Mycoses. 2005;48:165-171.

24. Vacheva-Dobrevski R, Kovachev S, Nacheva A, Stoev S, Vasilev N. Comparative study of itraconazole and fluconazole therapy in vaginal candidosis. Akush Ginekol (Sofiia). 2004;43:20-3.

25. Pitsouni E, Iavazzo C, Falagas ME. Itraconazole vs fluconazole for the treatment of uncomplicated acute vaginal and vulvovaginal candidiasis in nonpregnant women: a metaanalysis of randomized controlled trials. Am J Obstet Gynecol. 2008;198:153-60.

26. Hu Y, Hu Y, Lu Y, Huang S, Liu K, Han X, et al. A case Report of penile infection caused by fluconazole- and terbinafine-resistant Candida albicans. Mycopathologia. 2017;182:397-402.

27. Niwa T, Imagawa Y, Yamazaki H. Drug interactions between nine antifungal agents and drugs metabolized by human cytochromes P450. Curr Drug Metab. 2014;15:651-79.

Copyright by Alexandro Bonifaz, et al. This is an open-access article distributed under the terms of the Creative Commons Attribution License, which permits unrestricted use, distribution, and reproduction in any medium, provided the original author and source are credited.

Source of Support: Nil, Conflict of Interest: None declared. 